\title{
Hepatitis B virus $X$ protein influences enrichment profiles of H3K9me3 on promoter regions in human hepatoma cell lines
}

\author{
Di-Yi Wang ${ }^{1}$, Shu-Hong An ${ }^{2}$, Lei Liu ${ }^{1}$, Shan-Shan Bai ${ }^{3}$, Kai-Xiang Wu${ }^{1}$, Rong Zhu ${ }^{4}$, \\ Zhao-Jin Wang ${ }^{2}$ \\ ${ }^{1}$ Department of Pathology, Affiliated Hospital of Taishan Medical University, Taian 271000, China \\ ${ }^{2}$ Department of Human Anatomy, Taishan Medical University, Taian, 271000, China \\ ${ }^{3}$ Department of Pathology, The First people's Hospital of Taian, Taian, 271000, China \\ ${ }^{4}$ Department of Pathology, Shanghai Medical College, Fudan University, Shanghai 200032, China \\ Correspondence to: Zhao-Jin Wang, email: zjwang@tsmc.edu.cn \\ Keywords: hepatitis B virus X protein, histone H3 lysine 9 trimethylation, gene promoters, hepatocellular carcinoma \\ Received: May 10, $2016 \quad$ Accepted: October 12, $2016 \quad$ Published: October 19, 2016
}

\section{ABSTRACT}

We previously showed that hepatitis B virus (HBV) X protein (HBX) could promote the trimethylation of histone $\mathrm{H} 3$ lysine 9 ( $\mathrm{H} 3 \mathrm{~K} 9 \mathrm{me} 3)$ to repress tumor suppressor genes in hepatocellular carcinoma (HCC). In this work, we analyze 23,148 human promoters using ChIP-chip to determine the effects of HBX on H3K9me3 enrichments in hepatoma cells with transfection of $\mathrm{HBX}$-expressing plasmid. Immunohistochemistry for HBX and H3K9me3 was performed in 21 cases of HBV-associated HCC tissues. We identified that $\mathrm{H} 3 \mathrm{~K} 9 \mathrm{me} 3$ immunoreactivity was significantly correlated with HBx staining in HCC tissues. ChIP-chip data indicated that HBX remarkably altered promoter enrichments of $\mathrm{H} 3 \mathrm{~K} 9 \mathrm{me} 3$ in hepatoma cells. We identified 25 gene promoters, whose $\mathrm{H} 3 \mathrm{~K} 9 \mathrm{me} 3$ enrichments are significantly altered in hepatoma cells transfected HBx-expressing plasmid, including 19 gaining $\mathrm{H} 3 \mathrm{~K} 9 \mathrm{~m} 3$, and six losing this mark. Most of these genes have not been previously reported in HCC, and BTBD17, MIR6089, ZNF205-AS1 and ZP1 have not previously been linked to cancer; only two genes (DAB2IP and ZNF185) have been reported in HCC. Genomic analyses suggested that genes with the differential $\mathrm{H} 3 \mathrm{~K} 9 \mathrm{me} 3$ enrichments function in diverse cellular pathways and many are involved in cancer development and progression.

\section{INTRODUCTION}

Hepatitis B virus (HBV) chronic infection has been comfirmed as a major risk factor in the development of hepatocellular carcinoma (HCC) $[1,2]$. Hepatitis B virus $\mathrm{X}$ protein $(\mathrm{HBx})$, the product of the $\mathrm{HBV}$ gene HBX, is a pivotal factor in regulating gene transcription, participating in cell signaling, controlling cell proliferation, and immune response [2-4].

Histone modifications are essential and crucial for organizing the nuclear architecture, with subsequent effects on the regulation of gene transcription $[5,6]$. Several studies have indicated that HBx is involved in the tumorigenesis of $\mathrm{HCC}$ via histone modifications $[4,6]$. Histone $\mathrm{H} 3$ lysine 9 trimethylation $(\mathrm{H} 3 \mathrm{~K} 9 \mathrm{me} 3)$ is associated with gene silencing and serves as mechanism to inactivate tumor suppressor genes in various cancers [7-9]. Our previous studies demonstrated that $\mathrm{HBx}$ could promote both $\mathrm{H} 3 \mathrm{~K} 9$ trimethylation and DNA hypermethylation on the p16 promoter in hepatocarcinogenesis $[10,11]$. These findings implicated that $\mathrm{HBx}$-mediated $\mathrm{H} 3 \mathrm{~K} 9 \mathrm{me} 3$ might be involved in the tumor suppressor gene silencing in carcinogenesis. However, the effects of $\mathrm{HBx}$ on $\mathrm{H} 3 \mathrm{~K} 9 \mathrm{me} 3$ enrichment profiles on gene promoters were not fully understood.

Chromatin immunoprecipitation (ChIP) combined with genome tiling arrays (ChIP-chip) analysis is useful to determine epigenetic alterations in the genome $[12,13]$. cDNA microarray has been extensively used as a convenient and cost-effective technique for analyzing gene expression signatures. In the present study, we adopted ChIP-chip technology to profile and compare variations of $\mathrm{H} 3 \mathrm{~K} 9 \mathrm{me} 3$ enrichments on gene promoters in hepatoma cells with or without transfection of HBx-expressing plasmid to gain a better understanding of pathogenic mechanisms in HBV-associated HCC. 


\section{RESULTS}

\section{Expression of $\mathrm{HBx}$ and $\mathrm{H3} \mathrm{K} 9 \mathrm{me} 3$ in $\mathrm{HBV}$ - associated HCC tissues}

The expression of $\mathrm{HBx}$ and $\mathrm{H} 3 \mathrm{~K} 9 \mathrm{me} 3$ in HBV-associated HCC tissue was determined by immunohistochemistry. HBx immunoreactivity displayed distinctive cytoplasmic staining (Figure 1A, A1) and $\mathrm{H} 3 \mathrm{~K}$ 9me3 staining was observed strongly in the nucleus in $\mathrm{HCC}$ and adjacent non-cancerous tissues (Figure 1B, B1). Immunohistochemistry analysis showed that $\mathrm{H} 3 \mathrm{~K} 9$ me3 expression and $\mathrm{HBx}$ staining in $\mathrm{HCC}$ were significantly higher than those in adjacent non-cancerous tissues (Figure 1C; $P<0.05$ ). Pearson analysis showed that expression levels of $\mathrm{H} 3 \mathrm{~K} 9 \mathrm{me} 3$ were significantly correlated with $\mathrm{HBx}$ staining in $\mathrm{HBV}$-associated HCC tissues (Figure 1D, $\mathrm{r}=0.748, P<0.05$ ).

\section{$\mathrm{HBx}$ increased the level of $\mathrm{H} 3 \mathrm{~K} 9 \mathrm{me} 3$ in hepatoma cells}

HepG2 and SMMC-7721 cell lines were transfected with $\mathrm{HBx}$-expressing plasmid, and protein levels of $\mathrm{HBx}$ and H3K9me3 were detected by western blot analysis. No immunoblots for HBx was detected in both cell lines with control plasmid. The expression of HBx was detected at $24 \mathrm{~h}$, significantly increased at $48 \mathrm{~h}$ in both cell lines after transfection of $\mathrm{HBx}$-expressing plasmid (Figure 2A). We found that HBx could increase H3K9me3 levels in both cell lines, consistent with our previous study [11]. Pearson analysis showed that $\mathrm{H} 3 \mathrm{~K} 9 \mathrm{me} 3$ levels were significantly correlated with HBx protein expression (Figure 2A, $r=$ $0.791, P<0.05)$.

\section{HBx influenced H3K9me3 enrichment profiles on promoters in hepatoma cells}

To assess the enrichment pattern of HBx-mediated $\mathrm{H} 3 \mathrm{~K} 9 \mathrm{me} 3$ on regulatory regions of genes, we used ChIPchip to map profiles of H3K9me3 in HepG2 and SMMC7721 cell lines after transfection of HBx-expressing plasmid for $48 \mathrm{~h}$. Using a peak detection algorithm with a false discovery rate (FDR) of $\leq 0.05$, enrichment profiles were compared to those of transfection with control plasmid. We identified a total of 653 and $512 \mathrm{H} 3 \mathrm{~K} 9 \mathrm{me} 3$-enriched promoters in HepG2 and SMMC-7721 after transfection of HBx-expressing plasmid, 539 and 469 in controls respectively (Figure 2B, Supplementary Table S1). HBx induced a gain of $\mathrm{H} 3 \mathrm{~K} 9 \mathrm{me} 3$ on a substantial number of promoters in $\mathrm{HBx}$-expressing hepatoma cells, but did not prevent loss of this mark from others. About 27.1\% (146/539, HepG2) and 21.5\% (101/469, SMMC-7721) of H3K9me3-marked genes gained this mark "de novo" when transfected with $\mathrm{HBx}$-expressing plasmid (Figure 2B and 2C, Supplementary Table S1, S2 and S3). However,
$5.9 \%(32 / 539, \mathrm{HepG} 2)$ and $11.5 \%$ (54/469, SMMC-7721) of $\mathrm{H} 3 \mathrm{~K} 9 \mathrm{me} 3$-marked other genes lost this mark when transfected with $\mathrm{HBx}$-expressing plasmid (Figure 2B and 2D, Supplementary Table S1, S2 and S3).

In addition, average $\mathrm{H} 3 \mathrm{~K} 9 \mathrm{me} 3$ profiles are similar in controls and HBx-expressing hepatoma cells. Approximately $89 \%$ of $\mathrm{H} 3 \mathrm{~K} 9 \mathrm{me} 3$ sites were mapped to proximal regions of transcription start sites (TSSs) of RefSeq genes (from about -1000 bp to +400 bp of TSSs), including many sites located in downstream proximal regions of TSSs in HBx-expressing hepatoma cells or controls (Figure 2E). However, there were substantial increases in the numbers of $\mathrm{H} 3 \mathrm{~K} 9 \mathrm{me} 3$ enriched promoters in HBx-expressing hepatoma cells, compared to controls (Figure 2E, Supplementary Table S1).

\section{GO analysis of $\mathrm{H3K} 9 \mathrm{me} 3$ enrichments induced by $\mathrm{HBx}$}

To address the biological significance of enrichments in $\mathrm{HBx}$-mediated $\mathrm{H} 3 \mathrm{~K} 9$ me 3 revealed by ChIP-chip, we identified Gene Ontology (GO) terms enriched among these genes. We found $\mathrm{HBx}$-mediated enrichments in distinct terms for genes harboring this mark. Enriched GO terms for Biological process categories included G0 to G1 transition, leukocyte migration, DNA modification, and regulation of ARF protein signal transduction (Figure 3A; Supplementary Table S4). Enriched GO terms for Molecular function categories were mainly related to longchain fatty acid binding, insulin-like growth factor (IGF) receptor binding, ARF guanyl-nucleotide exchange factor activity (Figure 3A; Supplementary Table S5).

Among 109 genes annotated with the enriched GO term 'Biological process', 19 have a role in cell development, 18 in regulation of transcription from RNA polymerase II promoter, and 16 in defense response (Figure 3B, Supplementary Table S4). Among 101 genes annotated with the GO enriched term 'Molecular function', 64 have a role in protein binding (including BAIAP3, DAB2IP, FABP3, KCNN1, KLHL34, MBD1, SHANK1, SLC12A5, and ZNHIT1), and 10 in sequence-specific DNA binding (Figure 3B, Supplementary Table S5). Most of genes are well-known oncogenes (e.g. BAIAP3, DAB2IP, and FABP3). Association with carcinogenesisregulated genes seems therefore to be a feature of $\mathrm{HBx}$ mediated $\mathrm{H} 3 \mathrm{~K} 9 \mathrm{me} 3$ enrichment, however, we also found association of $\mathrm{HBx}$-mediated $\mathrm{H} 3 \mathrm{~K} 9 \mathrm{me} 3$ with metabolic processes (e.g. GAPDHS, GCNT1, and SLC16A3), which to our knowledge has not been reported earlier.

\section{HBx affected H3K9me3 enriched genes involved in tumorigenesis}

To elucidate the role of putative $\mathrm{H} 3 \mathrm{~K} 9 \mathrm{me} 3$ target genes in the tumorigenesis of HCC, we compared expression profiles of $\mathrm{H} 3 \mathrm{~K} 9 \mathrm{me} 3$ enriched promoters that 

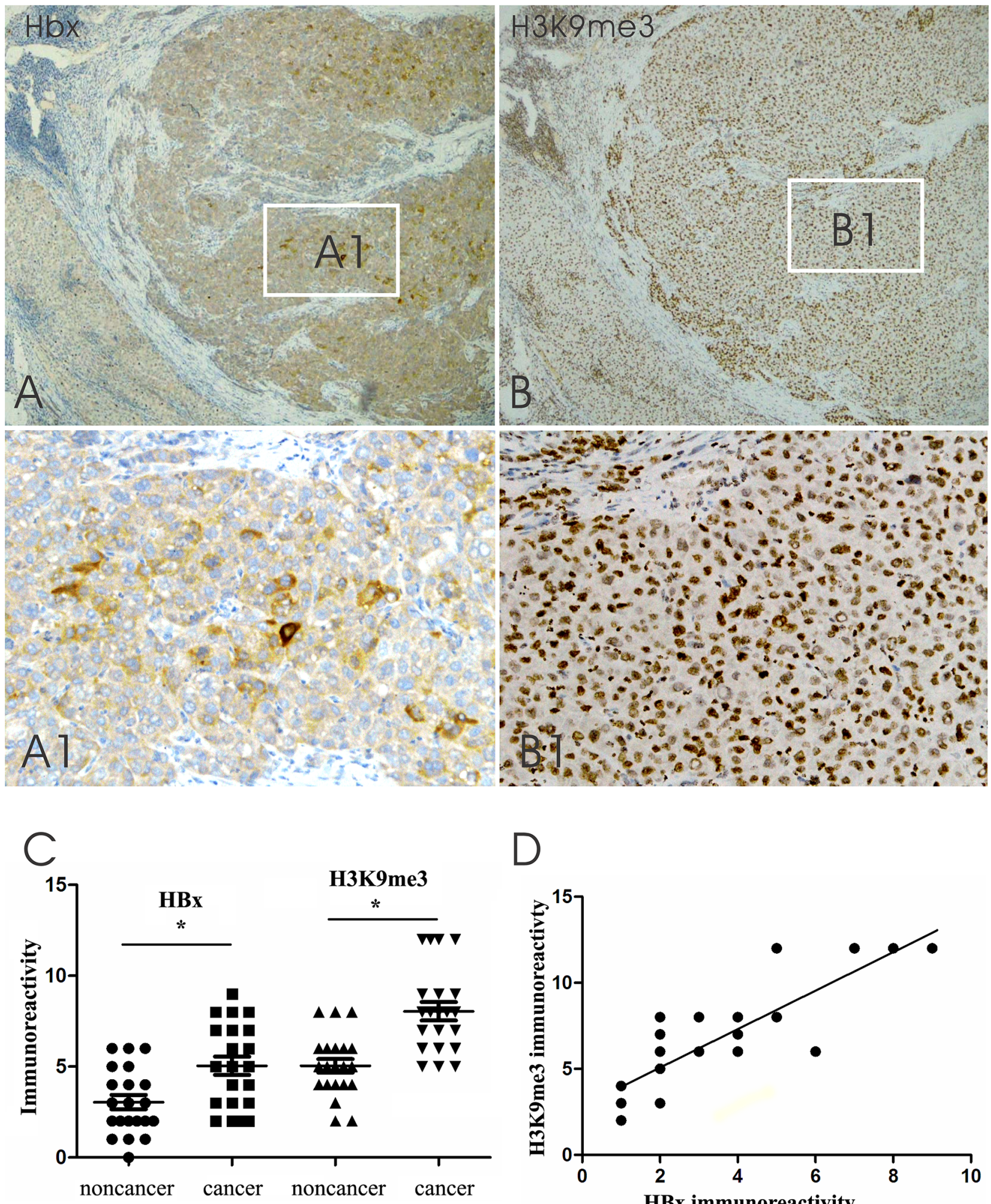

D

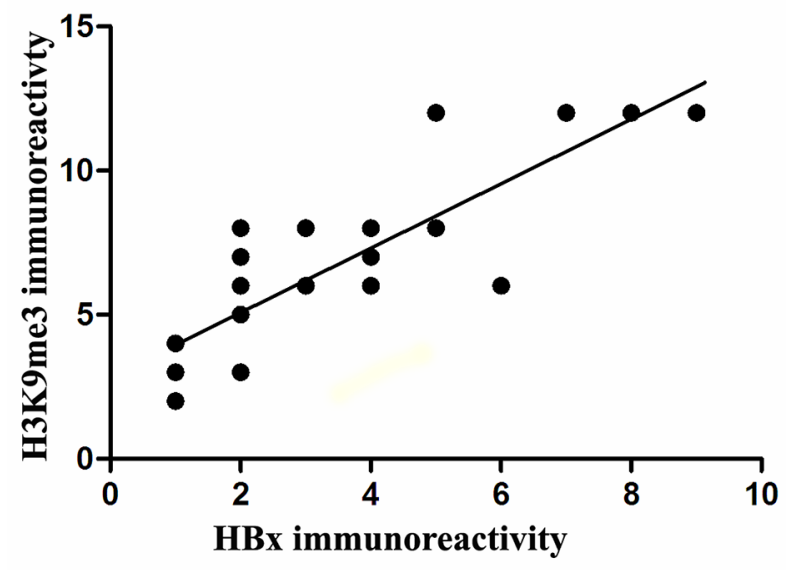

Figure 1: Representative $\mathrm{HBx}$ and $\mathrm{H3K} 9 \mathrm{me} 3$ immunoreactivity in corresponding region of parallel sections from the same HCC case. A. HBx immunoreactivity in HBV-associated HCC tissues and adjacent non-cancerous hepatic tissues. A1. Cytoplasmic HBx staining in the inset of A. B. H3K9me3 staining in the corresponding region of parallel section from the same $\mathrm{HCC}$ case. B1. Nuclear H3K9me3 staining in the inset of B (original magnifications $\times 40$ [A and B], $\times 200$ [A1 and B1]). C. Graph showing HBx and $\mathrm{H} 3 \mathrm{~K} 9 \mathrm{me} 3$ immunoreactivity in $\mathrm{HCC}$ tissues and adjacent noncancerous hepatic tissues. Immunoreactivity was calculated by multiplying the percentage and intensity scores. Data indicate mean \pm SEM. $* P<0.05$. D. Graph showing a positive correlation between HBx and H3K9me3 expression (Pearson analysis, $\mathrm{r}=0.748, P<0.05$ ). 
A
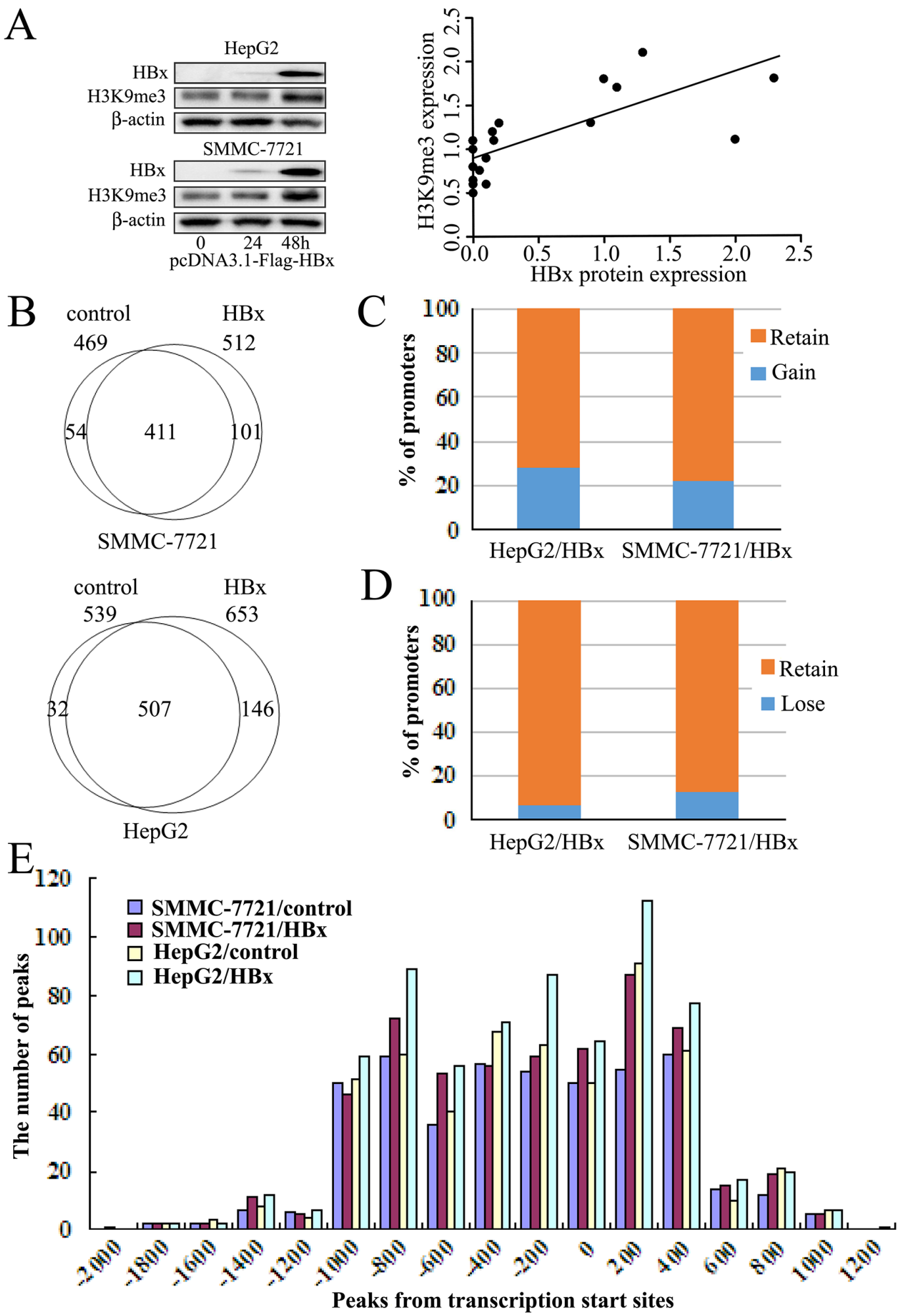

Figure 2: Alterations of H3K9me3 enriched promoters following HBx-expressing plasmid transfected into hepatoma cell lines for 48h. A. Western blot analysis of HBx and H3K9me3 expression in SMMC-7721 and HepG2 cell lines with transfection of HBx-expressing plasmid. Representative histograms showing a positive correlation between HBx and H3K9me3 expression in hepatoma cells after transfection of HBx-expression plasmid (Pearson analysis, $\mathrm{r}=0.791, P<0.05$ ). B. Venn diagraman analysis of gene promoters with enriched H3K9me3 in controls and HBx-expressing hepatoma cell lines. C. Percentage of genes with H3K9me3 that gains this mark "de novo" in HBx-expressing hepatoma cell lines. D. Percentages of genes that retain or lose H3K9me3 in HBx-expressing hepatoma cell lines, compared to controls. E. Distribution of H3K9me3 ChIP-chip peaks relative to gene TSSs. Shown are H3K9me3 peak frequencies relative to the distance from the nearest annotated TSS. 


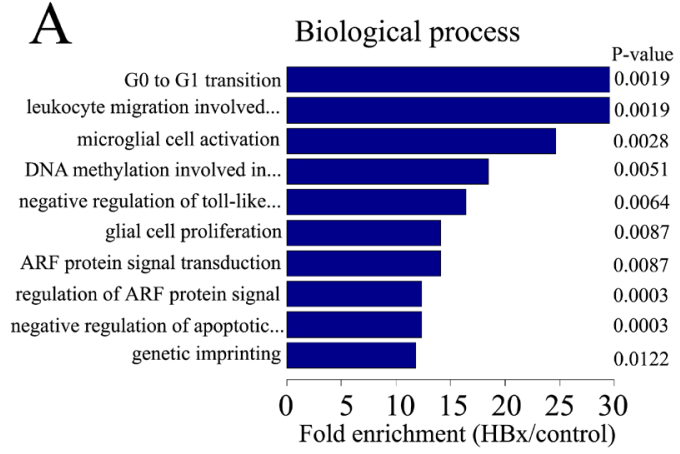

B

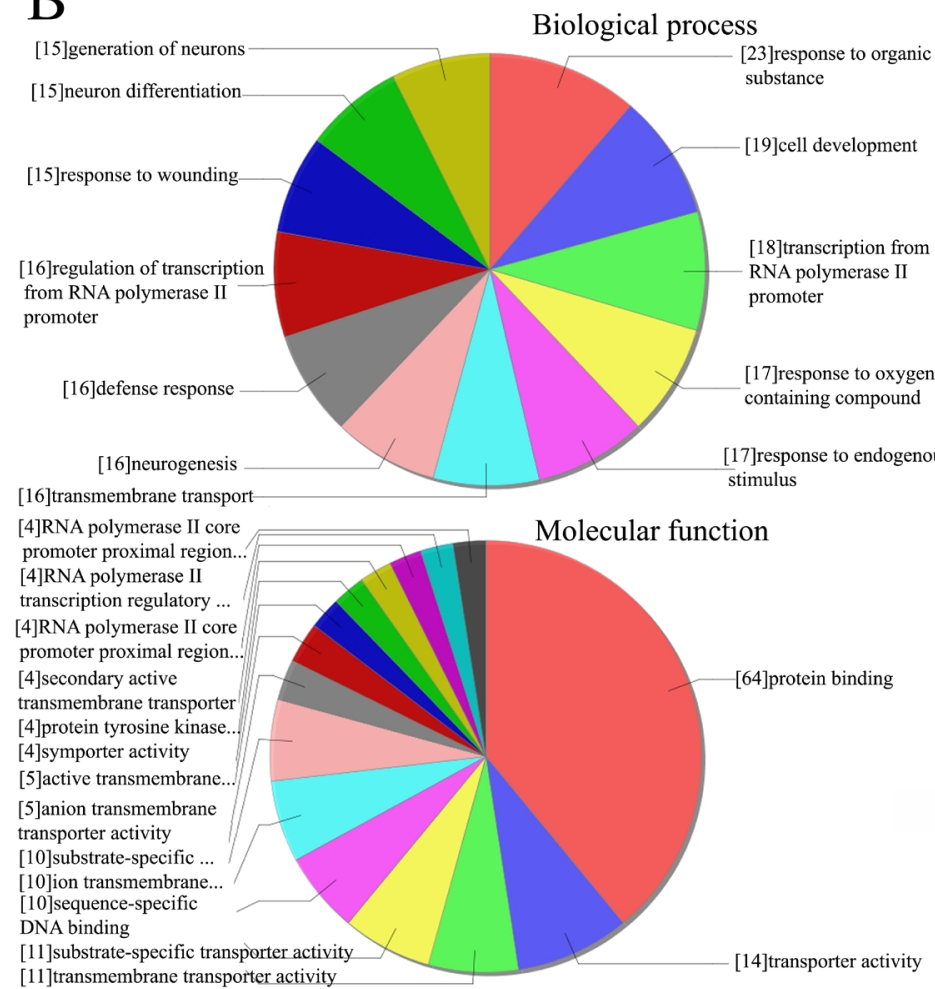

Molecular function

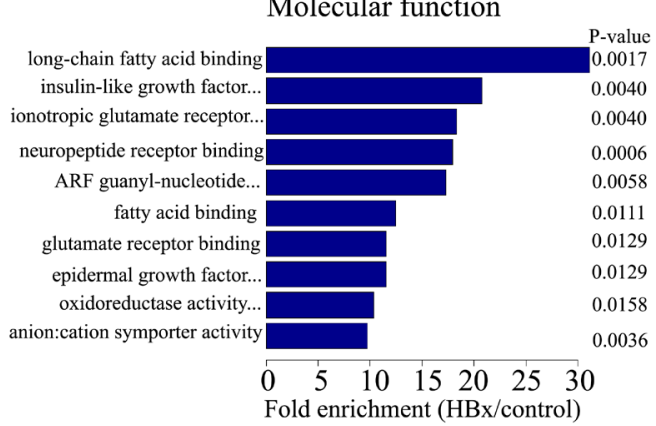

C
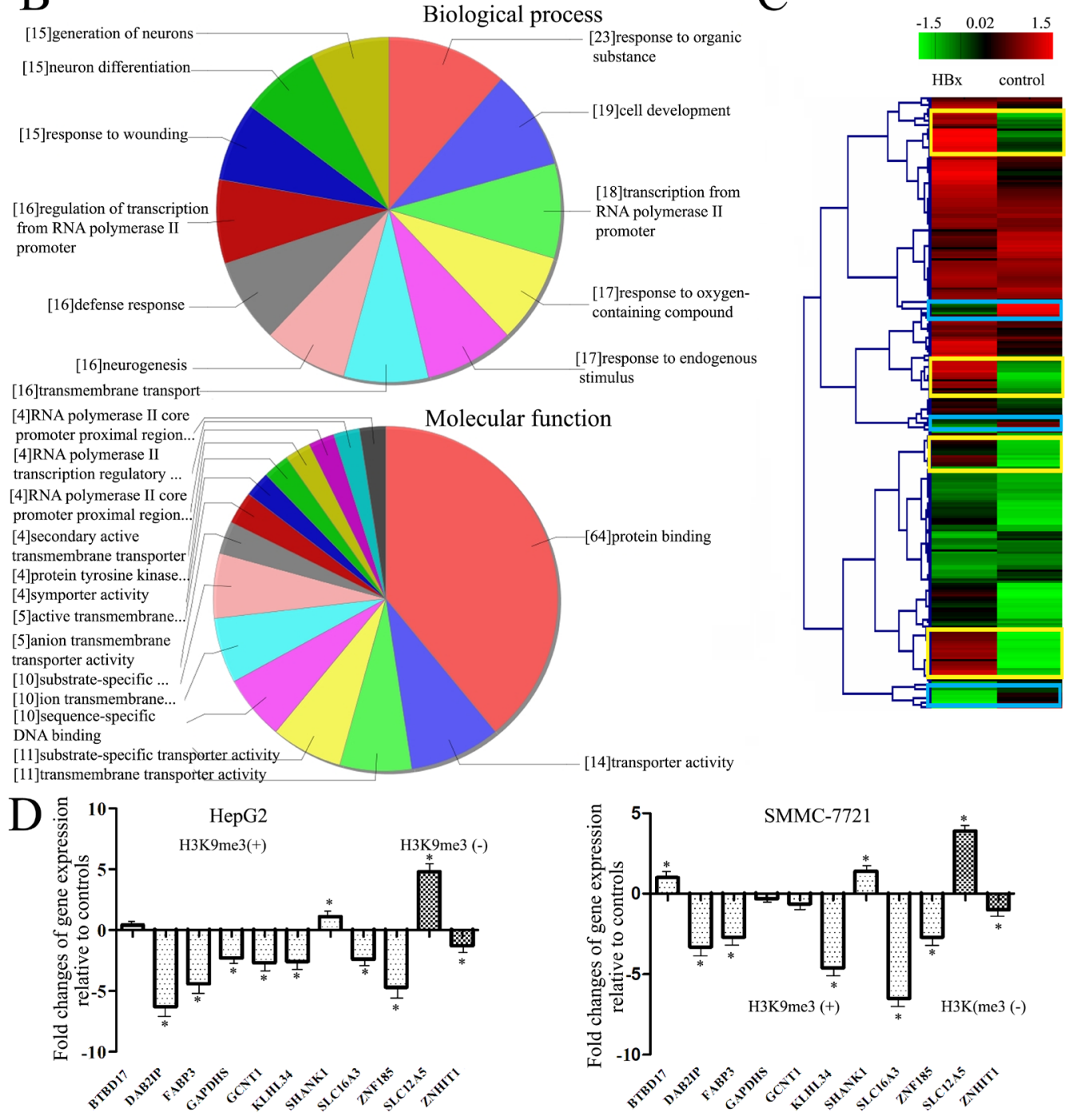

Figure 3: Enriched GO terms among genes which promoters were altered by HBx. A. Shown are the Biological process or Molecular functional categories of enriched genes after transfection of HBx-expressing plasmid, compared with controls. Bar plots show the top ten fold enrichment values of the significant enrichment terms. B. Further analysis of genes enriched the GO terms linked to the Biological process or Molecular functional categories of enriched genes after transfection of HBx-expressing plasmid, compared with controls. Charts show the top ten counts of the significant enrichment terms. C. Representative example of Hierarchical cluster analysis of data between HBx-expressing hepatoma cells and empty-controls. Yellow squares indicate genes with a greater H3K9me3 enrichment in HBx expressing hepatoma cells relative to controls; blue squares indicate genes with a lower H3K9me3 enrichment in HBx-expressing hepatoma cells relative to controls. D. Quantitative RT-PCR analysis for differences in expression levels of H3K9me specific target genes between HBx-expressing hepatoma cells and controls in the subset of genes gaining or losing H3K9me3 on their promoters. Results were calculated by normalizing to GAPDH in the same sample with the $\triangle \mathrm{Ct}$ method. Changes in relative levels of gene mRNAs expressed as folds of controls. All values were mean \pm SEM. $* P<0.05(\mathrm{n}=3)$. 
were differentially regulated in our experiments in controls and HBx-expressing cells. Genes enriching H3K9me3 mark were hierarchically clustered dependent on the gene H3K9me3 enrichment features of control against HBxexpressing cells (Figure 3C). The cluster of genes with higher $\mathrm{H} 3 \mathrm{~K} 9 \mathrm{me} 3$ enrichments in $\mathrm{HBx}$-expressing cells than in controls included DAB2IP, FABP3, SHANK1, and ZNF185. H3K9me3 losing genes included genes such as ZNHIT1 and SLC12A5. We identified nineteen H3K9me3 gaining genes and six H3K9me3 losing genes (Table 1) in both HepG2 and SMMC-7721 cell lines with transfection of HBx-expressing plasmid compared with controls. Most of these genes have not been previously reported in HCC, and BTBD17, MIR6089, ZNF205-AS1 and ZP1 have not previously been linked to cancer; only two genes (DAB2IP and ZNF185) have been reported in HCC. This clustering analysis of microarray data will indicate that the $\mathrm{HBx}-$ dependent transcriptional regulation is closely associated with the development of HCC.

\section{$\mathrm{HBx}$ altered the gene expression in hepatoma cells}

We next addressed the impact of gain or loss of $\mathrm{H} 3 \mathrm{~K} 9 \mathrm{me} 3$ induced by $\mathrm{HBx}$ on gene expression. We selected a subset of genes annotated with the GO terms enriched among genes gaining or losing H3K9me3 (at least 4-fold change), and assessed their expression at $48 \mathrm{~h}$ after transfection of $\mathrm{HBx}$-expressing plasmid by qRT-PCR. Results showed that most of genes gaining H3K9me3 became significantly downregulated, and genes losing this mark were significantly upregulated compared to controls (Figure 3D). However, altered H3K9me3 on promoters did not have a pronounced effect on some gene expression.

\section{DISCUSSION}

In the present study, we used a ChIP-chip microarray, which enables the genome-wide identification of binding sites of transcription factors and chromatin regulators $[12,13]$, and examined the influence of $\mathrm{HBx}$ on $\mathrm{H} 3 \mathrm{~K} 9 \mathrm{me} 3$ enrichment profiles on promoter regions to test the hypothesis that $\mathrm{H} 3 \mathrm{~K} 9 \mathrm{me} 3$ was associated with the pathogenesis of HBV-associated HCC $[10,11]$. We found that $\mathrm{H} 3 \mathrm{~K} 9 \mathrm{me} 3$ immunoreactivity levels were remarkably correlated to those of HBx in HCC tissues, and HBx was able to upregulate $\mathrm{H} 3 \mathrm{~K} 9 \mathrm{me} 3$ levels in hepatoma cell lines. CHIP-chip data showed that HBx could substantially increase the number of $\mathrm{H} 3 \mathrm{~K} 9 \mathrm{me} 3$-encriched promoters but did not prevent this mark loss on others, suggesting that $\mathrm{HBx}$ could alteration enrichment profiles of $\mathrm{H} 3 \mathrm{~K} 9 \mathrm{me} 3$ at promoters in a gene-specific manner.

In order to obtain insights into $\mathrm{H} 3 \mathrm{~K} 9 \mathrm{me} 3$ target gene function, GO analysis annotation were applied to the $\mathrm{H} 3 \mathrm{~K} 9 \mathrm{me} 3$ target gene pool. GO analysis showed
H3K9me3 enrichments on gene promoters were significantly influenced by transfection of $\mathrm{HBx}$-expressing plasmid. GO terms for Biological process categories included G0 to G1 transition, leukocyte migration, DNA modification, and regulation of ARF protein signal transduction. Most of genes are well-known oncogenes (e.g. BAIAP3, DAB2IP, FABP3, and ZNF185) [14-16]. On the other hand, GO terms for Molecular function categories were mainly related to long-chain fatty acid binding, IGF receptor binding, ARF guanyl-nucleotide exchange factor activity. Previous report showed that HBx could promote hepatic steatosis through up-expressing liver fatty acid binding protein [17]. However, fatty acid-binding protein was down-expressed in HCC and correlated with tumor differentiation and metastases [18]. The IGF system is involved in regulation of proliferation, differentiation, and apoptosis in the development of cancers [19]. ARF guanyl-nucleotide exchange factor plays a major role in mediating receptor endocytosis, Wnt signaling, and tumor metastases [20]. Therefore, alterations of this repressive mark induced by HBx could have severe consequences in hepatoma cell biology.

Among identified candidate genes, DAB2IP is a Ras GTPase-activating protein and plays a cancer suppressor role in tumorigenesis. The expression of DAB2IP is obviously low in HCC patients and inhibition of DAB2IP could contribute to HCC cell proliferation and invasion in vitro [14]. FABP3 may be involved in fatty acid transport, cellular differentiation and proliferation, signaling transduction, and gene transcription. Upregulation of FABP3 could result in mitochondrial dysfunction and lead to apoptosis [15]. ZNF185 may function as a tumor-suppressor protein by associating with the actin-cytoskeleton [16]. Study confirmed the down-regulation of ZNF185 gene in the drug-tolerant HCC cells with IFN-alpha treatment [21]. ZNHIT1 plays an important role in facilitating transcriptional activation during cell differentiation by a p38 MAPK-mediated signal mechanism [22]. In the present study, we found that $\mathrm{H} 3 \mathrm{~K} 9 \mathrm{me} 3$ enrichments on promoters of DAB2IP, FABP3, ZNF185 and ZNHIT1 displayed obvious alteration in HBx-expressing hepatoma cells, which would provide a better understanding of cell differentiation and proliferation abnormalities involved in the HBVassociated HCC patients.

SHANK1 may play tumor-associated roles in the development of malignant neoplasm [23]. The function of the sharpin (shank-associated RH domain interacting protein) may be involved in regulation of small GTPasemediated signaling pathways that may be required for NLRP3 (NLR family, pyrin domain containing 3) inflammasome activation and possibly plays active roles in immune cell proliferation and differentiation [24, 25]. Previous report showed that SLC16A3 might be involved in the tissue-specific transplantation antigen P35B (TSTA3)-activated network mediated immune response 
Table 1: HBx induced H3K9me3 enrich promoters in hepatoma cells identified by CHIP-chip

\begin{tabular}{|c|c|c|c|c|c|}
\hline Gene ID & Gene name & Peak region & Peak To TSS & $\begin{array}{c}\text { Fold } \\
\text { enrichment (vs. } \\
\text { control) }\end{array}$ & P-value \\
\hline \multicolumn{6}{|c|}{ Binding sites gain of $\mathrm{H3K} 9 \mathrm{me} 3$} \\
\hline NM_177947 & ARMCX3 & chrX: 100878290-100878762 & 76 & 2.55 & 0.0352 \\
\hline NM_001080466 & BTBD17 & chr17: $72357893-72359054$ & -515 & 5.83 & 0.0414 \\
\hline NR_033380 & CD99P1 & chrX: 2526402-2527078 & -426 & 2.01 & 0.0207 \\
\hline NM_032552 & DAB2IP & chr9: $124328114-124329861$ & -392 & 15.56 & 0.0003 \\
\hline NM_024045 & DDX50 & chr10: 70660864-70661331 & 64 & 3.65 & 0.0345 \\
\hline NM_004102 & FABP3 & chr1: $31845858-31846522$ & -267 & 16.11 & 0.0028 \\
\hline NM_014364 & GAPDHS & chr19: $36024175-36024695$ & 122 & 5.82 & 0.0366 \\
\hline NM_001097634 & GCNT1 & chr9:79056359-79056928 & 62 & 7.78 & 0.0028 \\
\hline NM_153270 & KLHL34 & chrX: 21676377-21677101 & -291 & 7.23 & 0.0350 \\
\hline NM_001204143 & MBD1 & chr18: $47808786-47809249$ & -880 & 2.07 & 0.0352 \\
\hline NR_106737 & MIR6089 & chrX: 2526402-2527355 & -352 & 2.23 & 0.0207 \\
\hline NM_001144856 & PLEKHG6 & chr12: 6419164-6419624 & -207 & 2.55 & 0.0200 \\
\hline NM_031915 & SETDB2 & chr13: $50018436-50019317$ & 448 & 2.04 & 0.0495 \\
\hline NM_016148 & SHANK1 & chr19: $51219710-51220370$ & 155 & 10.31 & 0.0055 \\
\hline NM_001206951 & SLC16A3 & chr17: $80185203-80185940$ & -93 & 4.82 & 0.0095 \\
\hline NM_032701 & SUV420H2 & chr19: $55850507-55851187$ & -373 & 2.26 & 0.0488 \\
\hline NM_001178113 & ZNF185 & chrX: 152086241-152086768 & 96 & 5.32 & 0.0273 \\
\hline NR_119385 & ZNF295-AS1 & chr21: 43441038-43441929 & -628 & 2.66 & 0.0287 \\
\hline NM_207341 & ZP1 & chr11 $60634137-60635106$ & -392 & 2.29 & 0.0371 \\
\hline \multicolumn{6}{|c|}{ Binding sites loss of $\mathrm{H} 3 \mathrm{~K} 9 \mathrm{me} 3$} \\
\hline NM_001031722 & ATAT1 & chr6: 30593317- 30593582 & -1164 & 2.24 & 0.0096 \\
\hline NM_001199096 & BAIAP3 & chr16: $1386416-1386676$ & 349 & 2.02 & 0.0232 \\
\hline NM_002248 & KCNN1 & chr19: $18060811-18061289$ & -1060 & 3.20 & 0.0076 \\
\hline NM_020708 & SLC12A5 & chr20: $44657861-44658166$ & 193 & 9.72 & 0.0076 \\
\hline NM_006062 & SMYD5 & chr2: 73440336- 73440814 & -790 & 3.03 & 0.0145 \\
\hline NM_006349 & ZNHIT1 & chr7: $100859752-100860221$ & -997 & 14.29 & 0.0019 \\
\hline
\end{tabular}

in hepatitis/cirrhotic tissues (HBV or HCV infection), such as T-cell homeostasis, neutrophil chemotaxis, interleukin-8 expression, immune response, and B-cell activation $[26,27]$. Our results showed that $\mathrm{HBx}$ could increase $\mathrm{H} 3 \mathrm{~K} 9 \mathrm{me} 3$ enrichment on genes of SHANK1 and SLC16A3, this might contribute to modulation of tumorimmune cell interactions.

Association with carcinogenesis-regulated genes seems therefore to be a feature of $\mathrm{HBx}$-mediated H3K9me3 enrichments, however, we also found association of $\mathrm{H} 3 \mathrm{~K} 9 \mathrm{me} 3$ with metabolic processes, such as genes GAPDHS, GCNT1 and SLC16A3, which to our knowledge has not been reported earlier in HBV-associated HCC. Study has found that GAPDH itself or GAPDHrelated proteins may be involved in lung tumorigenesis [28]. The expression of GCNT1 has strong correlation with tumorigenicity, development and metastasis in human prostate cancers [29, 30]. Furthermore, recent study has identified that DNA methylation of the SLC16A3 promoter is involved in the regulation of monocarboxylate transporter 4 (MCT4) in renal cancer resulting in cell apoptosis [31]. In this study, we have demonstrated that these $\mathrm{H} 3 \mathrm{~K} 9 \mathrm{me} 3$ enriched genes are not association with metabolic processes, but rather that they possibly play 
active roles in tumorigenesis. Since these gene expressions were most affected in cell proliferation and differentiation, signal transduction and transcription, it will be interesting to elucidate the effect of these genes on HBV-associated HCC tumorigenesis in future studies.

LncRNAs and miRNAs play a crucial role in the regulation of gene expression and modulation of signaling pathways, which contribute to the pathogenesis and progression of HCC [32]. Our results showed that HBxmediated $\mathrm{H} 3 \mathrm{~K} 9 \mathrm{me} 3$ enrichments were obviously upregulated on promoters of 1ncRNAs CD99P1, ZNF295AS1 and miRNA MIR6089. Previous study demonstrated that silencing CD99P1 inhibited proliferation and differentiation of lung fibroblasts [33]. ZNF205-AS1 and MIR6089, to our knowledge, have not been reported earlier in cancers.

In summary, we systematically evaluated $\mathrm{HBx}$ mediated H3K9me3 enrichments in hepatoma cells with transfection of $\mathrm{HBx}$-expressing plasmid and gained new insights into links between key genes and histone methylation induced by $\mathrm{HBx}$ in the development of HCC. Genomic analyses suggested that genes with the differential $\mathrm{H} 3 \mathrm{~K} 9 \mathrm{me} 3$ enrichments induced by HBx function in diverse cellular pathways and many are involved in cancer development and progression. These novel candidate genes showed here may become potential biomarkers or future therapeutic targets. Further investigations are needed to clarify roles of identified $\mathrm{H} 3 \mathrm{~K} 9 \mathrm{me} 3$ candidate genes in the pathogenesis of $\mathrm{HBV}$ associated HCC.

\section{MATERIALS AND METHODS}

\section{Cell culture and transfection}

Human heptoma cell lines (SMMC-7721 and HepG2) were purchased from the Cell Bank of the Chinese Academy of Sciences (Shanghai, China). HBx-expressing plasmid (pcDNA3.1-Flag-HBx) and control plasmid (pcDNA3.1) sequences, and transient transfection have been described previously $[10,11,34]$. Every cell line was transfected with $0.5 \mu \mathrm{g} /$ well of HBx-expressing plasmid or control plasmid for $48 \mathrm{~h}$ in 96 -well plates. HBx-transfected and empty-plasmid control cells are kept under identical culture conditions, expression analyses are performed at same passage numbers.

\section{Protein extraction and western blot analysis}

Cells were lysed, and protein was extracted as previously described $[10,11]$. Protein lysate from each sample was separated electrophoretically in sodium dodecyl sulfatepolyacrylamide gel, and then transferred to polyvinylidene fluoride (PVDF) membranes. Western blot analyses were performed with anti-HBx and antiH3K9me3 (Abcam, Cambridge, UK).

\section{Chromatin immunoprecipitation (ChIP), DNA labelling and array hybridization}

HepG2 and SMMC-7721 cell lines transfected with $\mathrm{HBx}$-expressing plasmid (2 biological replicates per cell lines) were chemically crosslinked, resuspended in lysis buffer, and sonicated fragments ranged in size from 200 to 1000 bp (Supplementary Figure S1A). Sonicated chromatin was resuspended in IP buffer and incubated with magnetic beads conjugated $\mathrm{H} 3 \mathrm{~K} 9 \mathrm{me} 3$ antibody (Abcam, Cambridge, UK). An un-enriched sample of DNA was treated in a similar manner to serve as input. The immunoprecipitated DNA was tested for enrichment of control loci by qPCR validation (primers and results are listed in Supplementary Table S6 and Supplementary Figure S1B, respectively). IgG control levels were below the threshold. The purified ChIP and input DNA was amplified and then fluorescently labeled using the NimbleGen Dual-Color DNA Labeling Kit, which is a single array designs that includes 23,148 gene promoter regions (from about $-1300 \mathrm{bp}$ to $+500 \mathrm{bp}$ of TSSs) totally covered by $\sim 180,000$ probes with approximately 210 bp spacing, dependent on the sequence composition of the region. The Cy5-ChIP and Cy3-input labelled DNA samples were co-hybridized to ArrayStar Human RefSeq Promoter Arrays, post hybridization washes were carried out and microarrays were scanned using Agilent Scanner G2505C.

\section{ChIP-chip data analysis}

Signal intensity data were extracted from scanned images using the NimbleScan v2.5 (Roche-NimbleGen). Log2 ChIP/Input ratios were scaled and centered around zero by subtracting the bi-weight mean for the $\log 2$ ratio values for all features on the array from each $\log 2$ ratio value. NimbleScan detects peaks by searching for 4 or more probes whose signals were above the specified cutoff values, ranging from $90 \%$ to $15 \%$, using a 1,000 bp sliding window. The cutoff values are a percentage of a hypothetical maximum, which is the mean +6 [standard deviation]. The ratio data is then randomized 20 times to evaluate the probability of "false positives". Each peak is then assigned a FDR score based on the randomization. The MA plots and box-plots were applied to assess the quality of raw data and effect of normalization (Supplementary Figure S1C and S1D). A correlation matrix was used to describe correlation between replicate experiments (Supplementary Figure S1E).

\section{Hierarchical cluster analysis for $\mathrm{H3K} 9 \mathrm{me3}$ enrichment promoters}

Hierarchical cluster analysis was performed for enriched genes selected by our experiments. Log2-ratio data for both genes gain and loss $\mathrm{H} 3 \mathrm{~K} 9 \mathrm{me} 3$ were clustered 
in a manner dependent on the gene $\mathrm{H} 3 \mathrm{~K} 9 \mathrm{me} 3$ enrichment features of HBx-expressing hepatoma cells and emptycontrols, using a hierarchical clustering by Cluster 3.0 software [35].

\section{Gene ontology analysis}

Gene ontology (GO) term enrichments within a target gene set were calculated using Gene Ontology (www.geneontology.org), which provides three structured networks of defined terms that describe gene product attributes. The $P$-value denotes the significance of GO Term enrichment in the differentially expressed gene list $(P<0.05$ was considered statistically significant).

\section{Quantitative reverse transcription-PCR (qRT-PCR) analysis}

The expression profiles of genes selected from enriched GO terms that showed the largest differences (Table 1) were assessed by qRT-PCR at $48 \mathrm{~h}$ after transfection of HBx-expressing plasmid. Total RNA was isolated from cells using TRIzol reagent (Invitrogen) according to the manufacturer's protocol. cDNA was synthesized using a cDNA synthesis kit (Invitrogen) according to the manufacturer's instructions. Primer sequences are listed in the Supplementary Table S6. qRTPCR was performed in triplicates by using a 7300 realtime PCR system (Applied Biosystems, Foster City, CA) according to the manufacturer's instructions.

\section{Immunohistochemistry analysis}

Immunohistochemical analyses were performed essentially as described before [10, 11]. Briefly, twentyone HBV positive HCC specimens were fixed in $10 \%$ buffered formalin. Parallel paraffin sections were stained with anti-HBx and anti-H3K9me3 (Abcam, Cambridge, UK), and then counterstained with hematoxylin. Immunoreaction scores were calculated by multiplying the percentage and intensity scores as our previously described [11].

\section{Statistical methods}

All results are expressed as mean \pm SEM. Statistical analysis was performed for the comparison of two groups in the microarray, immunoreactivity, and analysis of variance for comparisons was performed using the Student's t-test. Differences with $P<0.05$ were considered statistically significant. Correlation between $\mathrm{HBx}$ and H3K9me3 was analyzed using Pearson rank correlation.

\section{ACKNOWLEDGMENTS}

ChIP-chip experiments were performed by KangChen Bio-tech, Shanghai, China.

\section{CONFLICTS OF INTEREST}

No conflicts of interest are reported for any authors.

\section{GRANT SUPPORT}

This work was supported by National Natural Science Foundation of China (81371234) and Natural Science Foundation of Shandong Province (ZR2013HM032).

\section{REFERENCES}

1. Bosetti C, Turati F, La Vecchia C. Hepatocellular carcinoma epidemiology. Best Pract Res Clin Gastroenterol. 2014; 28 : 753-770.

2. Andrisani OM. Deregulation of epigenetic mechanisms by the hepatitis $\mathrm{B}$ virus $\mathrm{X}$ protein in hepatocarcinogenesis. Viruses. 2013; 5:858-8572.

3. Wang DY, Zou LP, Liu XJ, Zhu HG, Zhu R. Chemokine expression profiles of human hepatoma cell lines mediated by hepatitis B virus X protein. Pathol Oncol Res. 2016; 22:393-399.

4. Zhang XD, Wang Y, Ye LH. Hepatitis B virus X protein accelerates the development of hepatoma. Cancer Biol Med. 2014; 11:182-190.

5. Esteller M. Cancer epigenomics: DNA methylomes and histone-modification maps. Nat Rev Genet. 2007; 8:286-298.

6. Tian Y, Yang W, Song J, Wu Y, Ni B. Hepatitis B virus $\mathrm{X}$ protein-induced aberrant epigenetic modifications contributing to human hepatocellular carcinoma pathogenesis. Mol Cell Biol. 2013; 33:2810-2816.

7. Chiba T, Saito T, Yuki K, Zen Y, Koide S, Kanogawa N, Motoyama T, Ogasawara S, Suzuki E, OokaY, Tawada A, Otsuka M, Miyazaki M, et al. Histone lysine methyltransferase SUV39H1 is a potent target for epigenetic therapy of hepatocellular carcinoma. Int J Cancer. 2015; 136, 289-298.

8. Leszinski G, Gezer U, Siegele B, Stoetzer O, Holdenrieder S. Relevance of histone marks H3K9me3 and H4K20me3 in cancer. Anticancer Res. 2012; 32:2199-2205.

9. Gezer U, Ustek D, Yörüker EE, Cakiris A, Abaci N, Leszinski G, Dalay N, Holdenrieder S. Characterization of $\mathrm{H} 3 \mathrm{~K} 9 \mathrm{me} 3$ - and H4K20me3-associated circulating nucleosomal DNA by high-throughput sequencing in colorectal cancer. Tumour Biol. 2013; 34:329-336.

10. Zhu YZ, Zhu R, Shi LG, Mao Y, Zheng GJ, Chen Q, Zhu HG. Hepatitis B virus $X$ protein promotes hypermethylation of p16 (INK4A) promoter through upregulation of DNA methyltransferases in hepatocarcinogenesis. Exp Mol Pathol. 2010; 89:268-275.

11. Wang DY, Zou LP, Liu XJ, Zhu HG, Zhu R. Hepatitis $B$ virus $X$ protein induces the histone $\mathrm{H} 3$ lysine 
9 trimethylation on the promoter of p16 gene in hepatocarcinogenesis. Exp Mol Pathol. 2015; 99:399-408.

12. Komashko VM, Farnham PJ. 5-azacytidine treatment reorganizes genomic histone modification patterns. Epigenetics. 2010; 5:229-240.

13. Ostrup $\mathrm{O}$, Reiner AH, Aleström P, Collas P. The specific alteration of histone methylation profiles by DZNep during early zebrafish development. Biochim Biophys Acta. 2014; 1839:1307-1315.

14. Zhang X, Li N, Li X, Zhao W, Qiao Y, Liang L, Ding Y. Low expression of DAB2IP contributes to malignant development and poor prognosis in hepatocellular carcinoma. J Gastroenterol Hepatol. 2012; 27:1117-1125.

15. Zhou L, Jin J, Song G, Liu H, Liu M, Shi C, Qian L. $\alpha$-Lipoic acid ameliorates mitochondrial impairment and reverses apoptosis in FABP3-overexpressing embryonic cancer cells. J Bioenerg Biomembr. 2013; 45:459-466.

16. Zhang JS, Gong A, Young CY. ZNF185, an actincytoskeleton-associated growth inhibitory LIM protein in prostate cancer. Oncogene. 2007; 26:111-122.

17. Wu YL, Peng XE, Zhu YB, Yan XL, Chen WN, Lin X. Hepatitis $\mathrm{B}$ virus $\mathrm{X}$ protein induces hepatic steatosis by enhancing the expression of liver fatty acid binding protein. J Virol. 2015; 90:1729-1740.

18. Inoue M, Takahashi Y, Fujii T, Kitagawa M, Fukusato T. Significance of downregulation of liver fatty acid-binding protein in hepatocellular carcinoma. World J Gastroenterol. 2014; 20:17541-17551.

19. Tian D, Mitchell I, Kreeger PK. Quantitative analysis of insulin-like growth factor 2 receptor and insulin-like growth factor binding proteins to identify control mechanisms for insulin-like growth factor 1 receptor phosphorylation. BMC Syst Biol. 2016; 10:15.

20. Aizel K, Biou V, Navaza J, Duarte LV, Campanacci V, Cherfils J, Zeghouf M. Integrated conformational and lipidsensing regulation of endosomal ArfGEF BRAG2. PLoS Biol. 2013; 11:e1001652.

21. Wong N, Chan KY, Macgregor PF, Lai PB, Squire JA, Beheshti B, Albert M, Leung TW. Transcriptional profiling identifies gene expression changes associated with IFNalpha tolerance in hepatitis C-related hepatocellular carcinoma cells. Clin Cancer Res. 2005; 11:1319-1326.

22. Cuadrado A, Corrado N, Perdiguero E, Lafarga V, MuñozCanoves P, Nebreda AR. Essential role of p18Hamlet/ SRCAP-mediated histone H2A.Z chromatin incorporation in muscle differentiation. EMBO J. 2010; 29:2014-2025.

23. Jung J, Kim JM, Park B, Cheon Y, Lee B, Choo SH, Koh SS, Lee S. Newly identified tumor-associated role of human Sharpin. Mol Cell Biochem. 2010; 340:161-167.
24. Rodgers MA, Bowman JW, Fujita H, Orazio N, Shi M, Liang Q, Amatya R, Kelly TJ, Iwai K, Ting J, Jung JU. The linear ubiquitin assembly complex (LUBAC) is essential for NLRP3 inflammasome activation. J Exp Med. 2014; 211:1333-1347.

25. Redecke V, Chaturvedi V, Kuriakose J, Häcker H. SHARPIN controls the development of regulatory T cells. Immunology. 2016; 148:216-226.

26. Halestrap AP. The SLC16 gene family - structure, role and regulation in health and disease. Mol Aspects Med. 2013; 34:337-349.

27. Wang L, Huang J, Jiang M, Lin H. Tissue-specific transplantation antigen P35B (TSTA3) immune responsemediated metabolism coupling cell cycle to postreplication repair network in no-tumor hepatitis/cirrhotic tissues (HBV or HCV infection) by biocomputation. Immunol Res. 2012; 52:258-268.

28. Tatjewski M, Gruca A, Plewczynski D, Grynberg M. The proline-rich region of glyceraldehyde-3-phosphate dehydrogenase from human sperm may bind $\mathrm{SH} 3$ domains, as revealed by a bioinformatic study of low-complexity protein segments. Mol Reprod Dev. 2016; 83:144-148.

29. Chen Z, Gulzar ZG, St Hill CA, Walcheck B, Brooks JD. Increased expression of GCNT1 is associated with altered O-glycosylation of PSA, PAP, and MUC1 in human prostate cancers. Prostate. 2014; 74:1059-1067.

30. Sato T, Yoneyama T, Tobisawa Y, Hatakeyama S, Yamamoto H, Kojima Y, Mikami J, Mori K, Hashimoto Y, Koie T, Ohyama C. Core $2 \beta-1,6-\mathrm{N}$-acetylglucosaminyltransfer ase- 1 expression in prostate biopsy specimen is an indicator of prostate cancer aggressiveness. Biochem Biophys Res Commun. 2016; 470:150-156.

31. Fisel P, Kruck S, Winter S, Bedke J, Hennenlotter J, Nies AT, Scharpf M, Fend F, Stenzl A, Schwab M, Schaeffeler E. DNA methylation of the SLC16A3 promoter regulates expression of the human lactate transporter MCT4 in renal cancer with consequences for clinical outcome. Clin Cancer Res. 2013; 19:5170-5181.

32. Guerrieri F. Long non-coding RNAs era in liver cancer. World J Hepatol. 2015; 7:1971-1973.

33. Huang C, Yang Y, Liu L. Interaction of long noncoding RNAs and microRNAs in the pathogenesis of idiopathic pulmonary fibrosis. Physiol Genomics. 2015; 47:463-469.

34. Chen HY, Tang NH, Li XJ, Zhang SJ, Chen ZX, Wang $\mathrm{XZ}$. Transfection and expression of hepatitis $\mathrm{B}$ virus $\mathrm{X}$ gene and its effect on apoptosis in HL-7702 cells. World J Gastroenterol. 2004; 10:959-964.

35. de Hoon MJ, Imoto S, Nolan J, Miyano S. Open source clustering software. Bioinformatics. 2004, 20: 1453-1454. 